\title{
Targeted disruption of the murine cholecystokinin-1 receptor promotes intestinal cholesterol absorption and susceptibility to cholesterol cholelithiasis
}

\author{
David Q.-H. Wang, ${ }^{1,2}$ Frank Schmitz, ${ }^{3}$ Alan S. Kopin, ${ }^{3}$ and Martin C. Carey ${ }^{2}$ \\ ${ }^{1}$ Department of Medicine, Liver Center and Gastroenterology Division, Beth Israel Deaconess Medical Center, and 2Department of Medicine, \\ Gastroenterology Division, Brigham and Women's Hospital, Harvard Medical School and Harvard Digestive Diseases Center, Boston, Massachusetts, USA. \\ ${ }^{3}$ Molecular Pharmacology Research Center, Department of Medicine, Tufts New England Medical Center, Boston, Massachusetts, USA.
}

\begin{abstract}
Cholecystokinin (CCK) modulates contractility of the gallbladder, the sphincter of Oddi, and the stomach. These effects are mediated through activation of gastrointestinal smooth muscle as well as enteric neuron CCK-1 receptors (CCK-1Rs). To investigate the potential physiological and pathophysiological functions linked to CCK-1R-mediated signaling, we compared male WT and CCK-1R-deficient mice (129/SvEv). After 12 weeks on either a standard mouse chow or a lithogenic diet (containing $1 \%$ cholesterol, $0.5 \%$ cholic acid, and $15 \%$ dairy fat), small-intestinal transit time, intestinal cholesterol absorption, biliary cholesterol secretion, and cholesterol gallstone prevalence were compared in knockout versus WT animals. Analysis of mice on either the chow or the lithogenic diet revealed that CCK-1 $\mathrm{R}^{-/-}$animals had larger gallbladder volumes (predisposing to bile stasis), significant retardation of small-intestinal transit times (resulting in increased cholesterol absorption), and increased biliary cholesterol secretion rates. The elevation in bile cholesterol, coupled with a tendency toward gallbladder stasis (due to the absence of CCK-induced contraction), facilitates nucleation, growth, and agglomeration of cholesterol monohydrate crystals; this sequence of events in turn results in a significantly higher prevalence of cholesterol gallstones in the CCK-1R-null mice.
\end{abstract}

\section{Introduction}

Cholecystokinin (CCK) is a neuroendocrine peptide hormone that exhibits a variety of effects on many target tissues (1). Molecular, cellular, and physiologic approaches have been used to establish a functional role of the CCK-1 receptor (CCK-1R) on gallbladder smooth muscle, on pancreatic acini, at many levels in the gastrointestinal tract, and in the enteric nervous system and brain (2-4). Gallbladder contractile dysfunction in response to exogenously administered CCK or CCK octapeptide (CCK-8) has been observed in cholesterol gallstone patients $(5,6)$ and to a lesser extent $(7)$ in pigment gallstone patients compared with normal subjects (5-7). Interest in the potential role of the CCK-1R in gallbladder emptying was enhanced by recognition of the important pathogenic relationship between bile stasis and cholesterol gallstone formation $(8,9)$. Most notably, the association of impaired gallbladder emptying $(10,11)$ with increased nucleation of cholesterol-supersaturated bile, biliary sludge, and gallstones late in pregnancy $(12,13)$ affirms the crucial role of gallbladder stasis in the pathogenesis of cholesterol gallstones $(8,9)$. Nonetheless, divergent studies $(6,14)$ have suggested that two different gallstone subpopulations may exist, characterized by either defective or normal gallbladder motor function. Some $(5,15)$ have suggested that stasis is due to an intrinsic gallbladder defect, whereas others (5-7) have proposed that abnormalities of smooth muscle

Nonstandard abbreviations used: body weight (BW); CCK octapeptide (CCK-8); CCK-1 receptor (CCK-1R); cholecystokinin (CCK); cholesterol saturation index (CSI). Conflict of interest: The authors have declared that no conflict of interest exists.

Citation for this article: J. Clin. Invest. 114:521-528 (2004).

doi:10.1172/JCI200416801. contractile function are due to influences extrinsic to the gallbladder, including defective CCK stimulation or impaired postprandial CCK release. Earlier experiments using prairie dogs (16-18) and ground squirrels (18) demonstrated impaired smooth muscle contractility shortly after initiation of a lithogenic diet, a phenomenon presumed to be the result of biliary cholesterol supersaturation, with gallbladder mucosal absorption and deposition of cholesterol molecules in gallbladder muscle cells. Frequent gallbladder emptying through supraphysiological doses of CCK-8 administered i.v. restores gallbladder contractility (19) and prevents gallstones (usually the pigment variety) in subjects undergoing total parenteral nutrition (20). In the present work we have taken a different approach and used a mouse model to examine whether disruption of the Cck-1r gene influences biliary cholesterol secretion and cholesterol gallstone formation by affecting small-intestinal motility, a variable known to influence intestinal cholesterol absorption in humans (21). Our results demonstrate that absence of the CCK-1R in mice increases susceptibility to cholesterol gallstones via mechanisms that involve dysmotility of both the small intestine and the gallbladder.

\section{Results}

Gallbladder volumes, prevalence rates, and characteristics of gallstones. As found in the initial phenotypic study of the CCK-1R knockout compared to WT mice (22), body weight (BW) of both mouse strains was similar at the start of the study. We found here that BW remained similar between the two groups after they had been ingesting the lithogenic diet for 12 weeks. At this time point, gallbladder volumes in CCK-1 $\mathrm{R}^{-1}$ - mice were $46 \pm 25 \mu \mathrm{l}(n=37)$, significantly $(P<0.01)$ greater than the volume in WT mice of $26 \pm 18 \mu \mathrm{l}(n=26)$. Further- 
Table 1

Biliary lipid compositions of pooled gallbladder and individual hepatic bile specimens ${ }^{A}$

\begin{tabular}{|c|c|c|c|c|c|c|}
\hline Mouse & Mol\% Ch & Mol\% L & Mol\% BS & $\mathrm{L} /(\mathrm{L}+\mathrm{BS})$ & {$[T L](g / d l)$} & CSIB $^{B}$ \\
\hline \multicolumn{7}{|c|}{ Pooled gallbladder bile specimens } \\
\hline WT & 7.84 & 19.07 & 73.09 & 0.21 & 9.35 & 1.21 \\
\hline CCK-1R ${ }^{-1-}$ & 9.84 & 18.55 & 71.61 & 0.21 & 9.99 & 1.50 \\
\hline \multicolumn{7}{|c|}{ Individual hepatic bile specimens } \\
\hline \multicolumn{7}{|l|}{ WT } \\
\hline 1 & 6.66 & 16.53 & 76.81 & 0.18 & 1.61 & 1.81 \\
\hline 2 & 4.29 & 14.13 & 81.58 & 0.15 & 2.08 & 1.15 \\
\hline 3 & 3.86 & 12.29 & 83.84 & 0.13 & 2.80 & 1.10 \\
\hline 4 & 5.26 & 16.99 & 77.75 & 0.18 & 2.06 & 1.25 \\
\hline 5 & 7.08 & 21.18 & 71.74 & 0.23 & 1.55 & 1.47 \\
\hline 6 & 5.36 & 17.13 & 77.51 & 0.18 & 1.65 & 1.34 \\
\hline Mean \pm SD & $5.42 \pm 1.27$ & $16.37 \pm 3.02$ & $78.21 \pm 4.19$ & $0.18 \pm 0.03$ & $1.96 \pm 0.47$ & $1.35 \pm 0.26$ \\
\hline \multicolumn{7}{|l|}{ CCK-1R ${ }^{-/-}$} \\
\hline 1 & 5.93 & 16.16 & 77.92 & 0.17 & 2.02 & 1.46 \\
\hline 2 & 8.06 & 14.95 & 76.99 & 0.16 & 1.81 & 2.10 \\
\hline 3 & 7.54 & 13.27 & 79.19 & 0.14 & 1.96 & 2.10 \\
\hline 4 & 6.88 & 14.25 & 78.87 & 0.15 & 2.21 & 1.88 \\
\hline 5 & 5.98 & 16.84 & 77.18 & 0.18 & 1.92 & 1.43 \\
\hline 6 & 7.09 & 19.10 & 73.82 & 0.21 & 1.61 & 1.61 \\
\hline Mean \pm SD & $6.91 \pm 0.85^{c}$ & $15.76 \pm 2.08$ & $77.33 \pm 1.93$ & $0.17 \pm 0.02$ & $1.92 \pm 0.20$ & $1.76 \pm 0.31^{\mathrm{C}}$ \\
\hline
\end{tabular}

AValues were measured from pooled gallbladder bile specimens ( $n=20$ per group) and individual hepatic

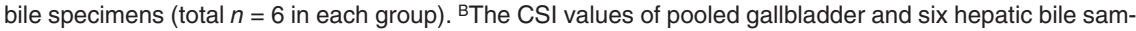
ples were calculated from the critical tables (23). ${ }^{C} P<0.05$ compared with hepatic bile samples of WT mice. CCK-1R, cholecystokinin-1 receptor; Ch, cholesterol; L, lecithin; BS, bile salt; [TL], total lipid concentration.

selected stones was pure cholesterol and constituted more than $99 \%$ of stone weight. Assessment of the frequency of gallstones in CCK-1R-/mice revealed that the number distribution fell between 1 and 3 in 46\% and between 4 and 6 in 19\%, while $5 \%$ of mice harbored more than 10 stones. In the WT mice, $19 \%$ formed 1-3 stones, 8\% produced 4-6 stones, and $4 \%$ had 7-9 stones. There were no differences in gallstone diameters between WT $(0.40 \pm 0.41 \mathrm{~mm})$ and CCK-1R $1 /$ mice $(0.42 \pm 0.33 \mathrm{~mm})$.

Lipid compositions of gallbladder and hepatic bile samples. Table 1 lists biliary lipid compositions of pooled gallbladder bile samples ( $n=20$ per group) and individual hepatic bile specimens $(n=6$ animals in each group) after 12 weeks of feeding the lithogenic diet. Compared with WT mice, CCK-1 $\mathrm{R}^{-/-}$mice displayed marked increases in cholesterol contents of both gallbladder and hepatic bile samples (Table 1). Moreover, the cholesterol saturation indexes (CSIs) of gallbladder bile specimens were clearly higher in $\mathrm{CCK}-1 \mathrm{R}^{-/-}$ mice $(\mathrm{CSI}=1.50)$ compared with WT

more, microscopy revealed that gallbladders from CCK-1 $\mathrm{R}^{-/-}$mice contained thick layers of mucus gel (100\%) interspersed with cholesterol monohydrate crystals (97\%) and aggregated liquid crystals (51\%). In contrast, gallbladders of WT mice tended to form thin layers of mucus gel (100\%), aggregated liquid crystals (81\%), and cholesterol monohydrate crystals (73\%). Gallstone prevalence in CCK$1 \mathrm{R}^{-/-}$mice $(70 \%)$ was significantly $(P<0.001)$ greater than that in WT mice $(31 \%)$. HPLC showed that the sterol extracted from randomly

\section{Figure 1}

Relative lipid compositions of bile specimens from CCK-1 $\mathrm{R}^{-/-}$and WT mice. Relative lipid compositions ( $\mathrm{mol}$ per $100 \mathrm{~mol}$ ) are plotted on partial condensed phase diagrams according to the approximate total lipid concentrations of the bile samples (A, $10.0 \mathrm{~g} / \mathrm{dl}$ for gallbladder bile; B, $2.0 \mathrm{~g} / \mathrm{dl}$ for hepatic bile; see Table 1). The one-phase micellar zone (at bottom) is enclosed by a solid curved line, and two solid and two dashed lines divide the phase diagram into regions a-e with different crystallization sequences (see ref. 24). (A) Lipid compositions of pooled gallbladder bile specimens ( $n=20$ per group) from the CCK-1 $\mathrm{R}^{-/}$(circle) and WT mice (square) fed the lithogenic diet for 12 weeks are located in a central three-phase area where, at equilibrium, bile samples are composed of cholesterol-saturated mixed micelles, solid cholesterol crystals, and liquid crystals, as observed by microscopy. (B) Analogous regions of the condensed phase diagram exhibit the same physical states at equilibrium as those in the phase diagram shown in $\mathbf{A}$; however, with decreases in total lipid concentration all crystallization pathways are shifted to the left and the micellar zone becomes smaller. These alterations generate a new condensed phase diagram with an enlarged region e. Lipid compositions of individual hepatic bile specimens ( $n=6$ per group) from both WT (squares) and CCK-1 $\mathrm{R}^{-/}$mice (circles) locate in region e, where, at equilibrium, the bile samples are composed of liquid crystals and saturated micelles, but no solid cholesterol monohydrate crystals are present. mice $(\mathrm{CSI}=1.21)(23)$. In addition, mean CSIs of hepatic bile specimens (Table 1$)$ were significantly $(P<0.05)$ higher in CCK-1R-/mice $(1.76 \pm 0.31)$ than in WT mice $(1.35 \pm 0.26)$.

Mean relative biliary lipid compositions of gallbladder bile are plotted on partial condensed phase diagrams for taurocholate-rich bile in Figure 1A, with the micellar phase boundary $(24,25)$ and cholesterol crystallization pathways insetted, for a total lipid concentration of $10.0 \mathrm{~g} / \mathrm{dl}$ (rounded off from the experimental value of $9.7 \mathrm{~g} / \mathrm{dl}$;

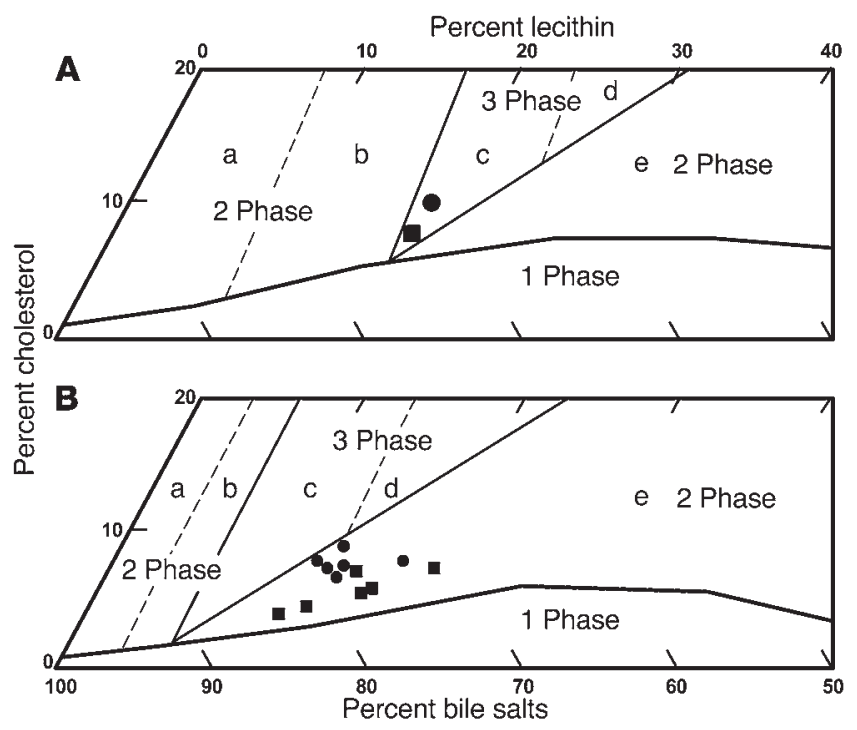




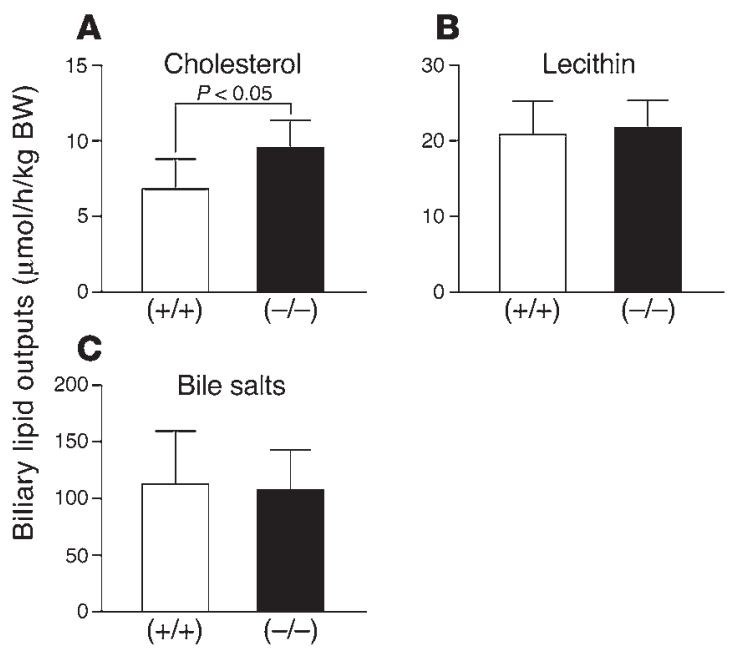

Figure 2

Total biliary lipid outputs $(\mu \mathrm{mol} / \mathrm{h} / \mathrm{kg} \mathrm{BW})$ during the first hour of interruption of the enterohepatic circulation in WT and CCK-1 ${ }^{-1-}$ mice ( $n=6$ per group) after feeding the lithogenic diet for 12 weeks. (A) The CCK-1 $\mathrm{R}^{-1-}$ mice displayed significantly higher biliary cholesterol outputs $(P<0.05)$ compared with the WT mice. However, biliary outputs of $(\mathbf{B})$ lecithin and $(\mathbf{C})$ bile salts were similar between the two sets of mice.

see Table 1) (24). Relative lipid compositions of gallbladder bile samples from CCK-1R $\mathrm{R}^{-/-}$mice fed the lithogenic diet plot in a central three-phase area denoted region c. By phase analysis, these bile samples are predicted to be composed of cholesterol monohydrate crystals, liquid crystals, and cholesterol-saturated mixed micelles at equilibrium $(24,25)$, as was observed experimentally. Gallbladder bile specimens of the WT mice fed the lithogenic diet also plotted in the central three-phase area; however, compared with the CCK-1R-/mice they contained appreciably lower amounts of cholesterol.

We plot in Figure 1B the relative lipid compositions of individual hepatic bile samples (from Table 1 ) in relation to an appropriate micellar phase boundary $(24,25)$ and cholesterol crystallization pathways for dilute $(2.0 \mathrm{~g} / \mathrm{dl})$ taurocholate-rich bile (rounded-off value for means of both sets of hepatic bile specimens in Table 1) (25). Relative lipid compositions plot within the crystallization pathway denoted region e, where only liquid crystals but never solid crystals phase separate at equilibrium (24). As observed in model (24) and native human bile samples (26), when total lipid concentrations decrease, equilibrium zones for all physical states and crystallization pathways shift markedly to the left, that is, to lower lecithin contents, and, in addition, the boundary of the micellar zone contracts downward. Our results show that relative lipid compositions from CCK-1 $\mathrm{R}^{-/-}$mice and WT controls plot in region e, with relative lipid compositions of hepatic bile samples from CCK-1 $\mathrm{R}^{-/-}$mice shifting upward significantly $(P<0.05)$, indicating increases in relative cholesterol contents (Table 1 ).

When gallbladder and hepatic bile samples from mice on a lithogenic diet were analyzed, all bile salt compositions from both WT and CCK-1 $\mathrm{R}^{-/}$mice were found to be taurine conjugated and displayed similar molecular species distributions. Because of the $0.5 \%$ cholic acid included in the lithogenic diet, taurocholate was, as expected $(27,28)$, the major bile salt $(75.4-76.9 \%)$, followed by diminishing relative proportions of tauro- $\beta$-muricholate (10.3-10.6\%) and taurodeoxycholate (8.1-9.7\%). Small concentrations of taurourso- deoxycholate (2.0-2.1\%), taurochenodeoxycholate (1.6-1.7\%), and tauro- $\omega$-muricholate $(0.8-0.9 \%)$ were also found. Hydrophobicity indexes (29) of gallbladder (0.03-0.06) and hepatic bile specimens (0.03-0.05) were identical between WT and CCK-1R $\mathrm{R}^{-/-}$mice.

Bile flow and biliary lipid secretion rates. Bile flow rates after 12 weeks of feeding the lithogenic diet (see Methods) were measured during the first hour after interruption of the enterohepatic circulation, thereby avoiding appreciable perturbation of the enterohepatic circulation of bile salts (27). In CCK-1R $/-$ mice, the bile flow rate $(69 \pm 10 \mu \mathrm{l} / \mathrm{min} / 100 \mathrm{~g} \mathrm{BW})$ was similar to that of the WT mice $(64 \pm 16 \mu \mathrm{l} / \mathrm{min} / 100 \mathrm{~g} \mathrm{BW})$. Figure 2 plots the individual biliary lipid outputs in WT and CCK-1 $\mathrm{R}^{-/-}$mice during the first hour of acute fistulation. A significant $(P<0.05)$ difference was observed only in biliary cholesterol secretion rates, which were $9.7 \pm 1.7 \mu \mathrm{mol} / \mathrm{h} / \mathrm{kg}$ BW in CCK-1R $-1-$ mice, and $6.8 \pm 2.1 \mu \mathrm{mol} / \mathrm{h} / \mathrm{kg} \mathrm{BW}$ in WT mice. No appreciable differences in lecithin or bile salt outputs (Figure 2) were noted; in CCK-1R $1 \mathrm{R}^{-/}$mice, these values were $21.9 \pm 3.0$ and $106.2 \pm 3.4$ $\mu \mathrm{mol} / \mathrm{h} / \mathrm{kg} \mathrm{BW}$, respectively, whereas in WT mice the values were $20.8 \pm 4.9$ and $113.0 \pm 4.7 \mu \mathrm{mol} / \mathrm{h} / \mathrm{kg} \mathrm{BW}$, respectively.

Volumes of the circulating bile salt pool were also identical between CCK-1R ${ }^{-/-}(2.4 \pm 0.3 \mu \mathrm{mol})$ and WT mice $(2.3 \pm 0.2 \mu \mathrm{mol})$. However, after factoring in each bile salt mass measured in pooled gallbladder bile samples, total bile salt pool sizes became appreciably greater in CCK-1 $\mathrm{R}^{-/-}$mice $(7.2 \mu \mathrm{mol})$ compared with WT mice $(5.4 \mu \mathrm{mol})$. This finding is consistent with the significantly enlarged fasting gallbladder volumes in CCK-1R $\mathrm{R}^{-/-}$mice compared with those of WT mice.

Intestinal cholesterol absorption by dual-isotope and cholesterol balance methods. Displayed in Figure 3 are values for percentage cholesterol absorption in mice ingesting chow as calculated from the plasma ratios of $\left[{ }^{14} \mathrm{C}\right]$ cholesterol and $\left[{ }^{3} \mathrm{H}\right]$ cholesterol 3 days after dosing $(30,31)$. Percentage cholesterol absorption values were significantly

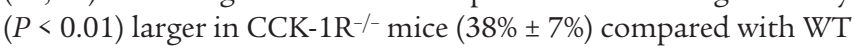
mice $(30 \% \pm 5 \%)$. Since chow contains trace cholesterol $(<0.02 \%)$, and since both CCK-1R $-1-$ and WT mice ate similar amounts of food (4.3-4.5 g/day), we calculated total cholesterol mass absorbed from the small intestine to be approximately $0.33 \mathrm{mg} /$ day in CCK-1 $\mathrm{R}^{-/}$ mice and approximately $0.26 \mathrm{mg} /$ day in WT mice. To demonstrate that this observation was sustained during feeding the lithogenic

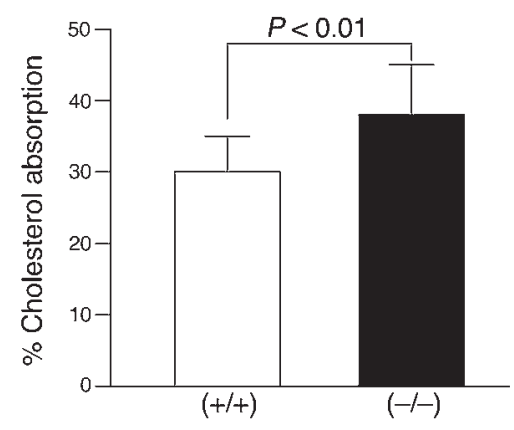

\section{Figure 3}

Percentage cholesterol absorption, as determined by the plasma dualisotope ratio method $(27,30,31)$ in chow-fed WT and CCK-1 $\mathrm{R}^{-/-}$mice ( $n=14$ per group). The CCK-1 $\mathrm{R}^{-1-}$ mice displayed significantly $(P<0.01)$ higher intestinal cholesterol absorption efficiencies compared with the WT mice (see details in Methods and Discussion). Because of dilution of radioisotopic cholesterol by the high-cholesterol diet, this method cannot be used to study percentage cholesterol absorption when mice are fed the lithogenic (gallstone-inducing) diet. However, as shown in Table 2, cholesterol mass balance studies are used under these conditions. 
Table 2

Cholesterol balance data in WT and CCK-1 $\mathrm{R}^{-1-}$ mice during ingestion of chow or lithogenic diet under metabolic steady-state conditions

\begin{tabular}{|c|c|c|c|c|c|}
\hline Mouse & $\begin{array}{l}\text { Cholesterol } \\
\text { intake } \\
\text { (mg/day) }\end{array}$ & $\begin{array}{c}\text { Biliary } \\
\text { cholesterol } \\
\text { (mg/day) }\end{array}$ & $\begin{array}{l}\text { Steroid } \\
\text { excretion } \\
\text { (mg/day) }\end{array}$ & $\begin{array}{c}\text { Absorbed } \\
\text { cholesterolA } \\
\text { (mg/day) }\end{array}$ & $\begin{array}{c}\text { Cholesterol } \\
\text { absorption } \\
\text { (\%) }\end{array}$ \\
\hline \multicolumn{6}{|l|}{ Chow diet } \\
\hline \multicolumn{6}{|l|}{ WT } \\
\hline 1 & 0.80 & 0.91 & 1.48 & 0.22 & 28.1 \\
\hline 2 & 0.83 & 1.29 & 1.83 & 0.28 & 34.3 \\
\hline 3 & 0.89 & 1.31 & 1.88 & 0.31 & 34.8 \\
\hline 4 & 0.81 & 1.34 & 1.91 & 0.24 & 29.8 \\
\hline 5 & 0.78 & 1.39 & 1.95 & 0.22 & 28.7 \\
\hline 6 & 0.78 & 1.18 & 1.78 & 0.18 & 23.4 \\
\hline 7 & 0.83 & 1.25 & 1.86 & 0.21 & 25.9 \\
\hline Mean \pm SD & $0.82 \pm 0.04$ & $1.24 \pm 0.16$ & $1.81 \pm 0.16$ & $0.24 \pm 0.04$ & $29.3 \pm 4.2$ \\
\hline \multicolumn{6}{|l|}{ CCK-1R ${ }^{-1-}$} \\
\hline 1 & 0.80 & 1.67 & 2.12 & 0.34 & 42.7 \\
\hline 2 & 0.88 & 1.23 & 1.68 & 0.43 & 48.5 \\
\hline 3 & 0.70 & 1.93 & 2.38 & 0.25 & 36.4 \\
\hline 4 & 0.77 & 1.94 & 2.42 & 0.28 & 36.6 \\
\hline 5 & 0.84 & 1.82 & 2.39 & 0.28 & 33.0 \\
\hline 6 & 0.79 & 1.43 & 1.95 & 0.27 & 34.8 \\
\hline 7 & 0.83 & 1.54 & 2.08 & 0.29 & 35.1 \\
\hline Mean \pm SD & $0.80 \pm 0.06$ & $1.65 \pm 0.27 c$ & $2.15 \pm 0.27 \mathrm{D}$ & $0.31 \pm 0.06^{\mathrm{D}}$ & $38.2 \pm 5.5^{c}$ \\
\hline \multicolumn{6}{|c|}{ Lithogenic diet } \\
\hline \multicolumn{6}{|l|}{ WT } \\
\hline 1 & 42.03 & 2.89 & 30.88 & 14.04 & 33.4 \\
\hline 2 & 41.30 & 1.59 & 26.66 & 16.23 & 39.3 \\
\hline 3 & 36.17 & 2.61 & 24.13 & 14.65 & 40.5 \\
\hline 4 & 36.93 & 1.58 & 26.47 & 12.04 & 32.6 \\
\hline 5 & 37.23 & 1.64 & 25.20 & 13.66 & 36.7 \\
\hline 6 & 47.47 & 2.32 & 35.31 & 14.48 & 30.5 \\
\hline 7 & 49.40 & 1.28 & 35.27 & 15.41 & 31.2 \\
\hline Mean \pm SD & $41.50 \pm 5.26^{E}$ & $1.99 \pm 0.61^{D}$ & $29.13 \pm 4.70^{\mathrm{E}}$ & $14.36 \pm 1.33^{\mathrm{E}}$ & $34.9 \pm 4.0^{\mathrm{F}}$ \\
\hline \multicolumn{6}{|l|}{ CCK-1R ${ }^{-/-}$} \\
\hline 1 & 43.93 & 3.25 & 24.65 & 22.54 & 51.3 \\
\hline 2 & 48.03 & 2.62 & 23.13 & 27.52 & 57.3 \\
\hline 3 & 38.07 & 3.54 & 22.92 & 18.69 & 49.1 \\
\hline 4 & 41.80 & 2.73 & 24.67 & 19.86 & 47.5 \\
\hline 5 & 43.60 & 2.90 & 27.92 & 18.57 & 42.6 \\
\hline 6 & 45.97 & 2.91 & 28.10 & 20.78 & 45.2 \\
\hline 7 & 51.73 & 3.10 & 31.19 & 23.64 & 45.7 \\
\hline Mean \pm SD & $44.73 \pm 4.40^{G}$ & $3.01 \pm 0.32^{\mathrm{G}, \mathrm{H}}$ & $26.09 \pm 3.06^{G}$ & $21.66 \pm 3.20^{\mathrm{G}, \mathrm{I}}$ & $48.4 \pm 4.8^{\mathrm{G}, \mathrm{I}}$ \\
\hline
\end{tabular}

${ }^{A}$ Absorbed cholesterol was determined by subtracting the daily fecal neutral steroid output from the daily cholesterol intake and the daily biliary cholesterol output as measured by HPLC (31). BThe percentage cholesterol absorption was determined by the cholesterol balance analysis according to published methods (31). ${ }^{C} P<0.01$ compared with WT mice on chow. $\mathrm{D} P<0.05$ compared with WT mice on chow. EP $<0.00001$ compared with WT mice on chow. $\mathrm{F} P<0.001$ compared with WT mice on chow. GP $<0.00001$ compared with CCK-1R ${ }^{-1-}$ mice on chow. ${ }^{H} P<0.01$ compared with WT mice fed the lithogenic diet. ${ }^{I} P<0.001$ compared with WT mice fed the lithogenic diet.

diet, it became necessary to study cholesterol absorption in mice ingesting the diet high in cholesterol, cholic acid, and triglycerides, as used for the cholesterol gallstone prevalence study.

Table 2 summarizes the per diem results for cholesterol intake, biliary cholesterol outputs, fecal total neutral steroid excretion, absorbed cholesterol mass, and percentage cholesterol absorption in
CCK-1 $\mathrm{R}^{-1-}$ and WT mice in the metabolic steady state while on the lithogenic diet or ingesting chow. Basically, we found that CCK-1R $-1-$ and WT mice ingested similar amounts of food whether they were fed the chow or the lithogenic diet. On the chow $\operatorname{diet}(\sim 0.02 \%$ cholesterol), biliary cholesterol secretion rates in CCK-1R $1 \mathrm{R}^{-/-}$mice $(1.65 \pm 0.27$ $\mathrm{mg} /$ day $)$ were significantly $(P<0.01)$ higher than in the WT mice $(1.24 \pm 0.16 \mathrm{mg} /$ day). Because of their higher biliary cholesterol outputs, CCK-1R $\mathrm{R}^{-/}$ mice showed significantly $(P<0.05)$ augmented daily fecal neutral sterol excretion $(2.15 \pm 0.27$ $\mathrm{mg} /$ day) compared with WT mice $(1.81 \pm 0.16$ $\mathrm{mg} /$ day). Nonetheless, an input-output analysis revealed that the absorbed cholesterol mass in CCK- $1 \mathrm{R}^{-1-}$ mice $(0.31 \pm 0.06 \mathrm{mg} /$ day $)$ was significantly $(P<0.05)$ larger than in the WT mice $(0.24 \pm 0.04 \mathrm{mg} /$ day $)$. The calculated percentage cholesterol absorption in CCK- $1 \mathrm{R}^{-/-}$mice was $38.2 \% \pm 5.5 \%$, a value that is significantly $(P<0.01)$ greater than in WT mice $(29.3 \% \pm 4.2 \%)$, and both are essentially identical to the results obtained with the dual-isotope method (see earlier). While ingesting the lithogenic diet, CCK-1 $\mathrm{R}^{-/}$mice also secreted significantly $(P<0.01)$ more biliary cholesterol than the WT mice (Table 2). Furthermore, cholesterol mass absorbed from the small intestine was significantly $(P<0.001)$ greater in CCK-1R $\mathrm{R}^{-/}$ mice than in the WT mice, consistent with the latter excreting appreciably more neutral steroids in feces. As was clarified elsewhere (27), we note that compared with chow, cholesterol absorption efficiency measured by the mass balance method was increased significantly $(P<0.001)$ in both strains of mice fed the lithogenic diet, mostly as a result of the dietary cholic acid (27). Furthermore, with lithogenic-diet feeding, CCK-1R $-1-$ mice continued to display significantly $(P<0.001)$ higher percentage cholesterol absorption $(48.4 \% \pm 4.8 \%)$ compared to the WT mice $(34.9 \% \pm 4.0 \%)$. Taken together, these results suggest that CCK-1 $\mathrm{R}^{-/-}$ mice absorb more cholesterol from the small intestine than do WT mice, whether they are fed trace or high levels of dietary cholesterol (and cholic acid).

Small-intestinal transit times. Figure 4 depicts the distributions of radioactivity administered as $\left[{ }^{3} \mathrm{H}\right]$ sitostanol along the length of the small intestines of CCK-1 $1 \mathrm{R}^{-/-}$and WT mice while each strain was being fed chow (Figure 4A) or the lithogenic diet (Figure 4B) for 14 days. These data were obtained at exactly 30 minutes after intraduodenal instillation of the radioisotope dissolved in medium-chain triglyceride (see Methods and references therein). On the chow diet, distributions of radioactivity lengthwise throughout the small intestine were significantly $(P<0.01)$ different between the WT and CCK-1 $\mathrm{R}^{-/-}$mice, with peaks occurring between segments 8 and 15 in WT mice compared with segments 4 and 11 in CCK-1R $1 \mathrm{R}^{-/-}$mice. The geometric center (see Methods) of the $\left[{ }^{3} \mathrm{H}\right]$ sitostanol distribution profiles in the small 


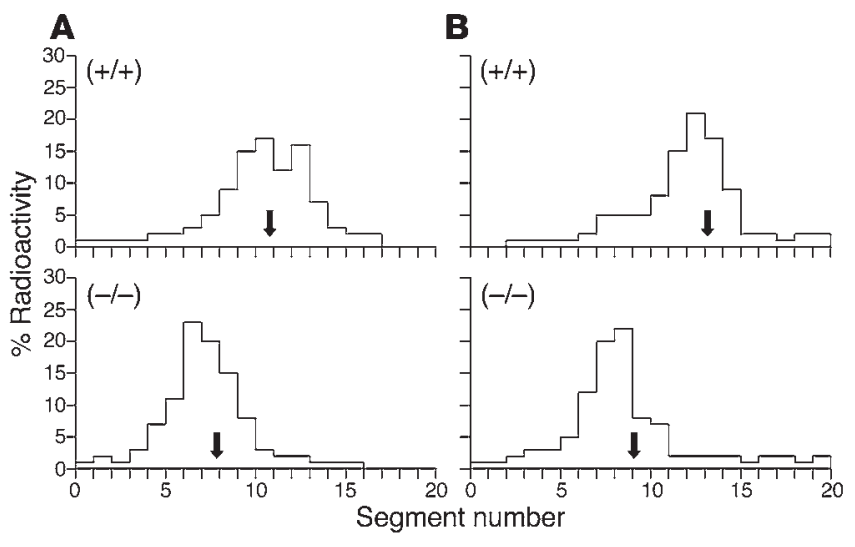

Figure 4

Small-intestinal transit rates in WT mice (top panels) and CCK-1 $\mathrm{R}^{-/-}$ mice (bottom panels) ingesting chow (A) or the lithogenic diet (B) for 14 days. Data were determined by the distribution of radioactivity at 30 minutes along the entire length of the small intestine after intraduodenal instillation of $\left[{ }^{3} \mathrm{H}\right]$ sitostanol dissolved in medium-chain triglyceride (30). Each bar shows the mean percentage of radioactivity in each segment for $n=13$ mice per group. Segments 1-20 represent evenly divided portions from the most proximal to the most distal parts of the small intestine placed on a 50-cm ruler (see Methods). (A) Arrows indicate the geometric center, which is significantly $(P<0.001)$ shorter in chow-fed CCK-1 $\mathrm{R}^{-/-}$mice, indicating significantly slower small-intestinal transit times (geometric center $=7.8 \pm 0.8$ ) compared with the WT mice (geometric center $=10.8 \pm 1.0$ ). (B) Upon ingesting the lithogenic diet, CCK-1 $\mathrm{R}^{-/-}$mice continue to display significantly slower smallintestinal transit times (geometric center $=9.1 \pm 1.5$ ) compared with the WT mice (geometric center $=13.3 \pm 2.0$ ).

intestine of WT mice was $10.8 \pm 1.0$, whereas in CCK- $1 \mathrm{R}^{-/-}$mice the value was $7.8 \pm 0.8$. This significant $(P<0.001)$ difference was again consistent with slower small-intestinal transit times in CCK-1 $\mathrm{R}^{-1-}$ mice. Although there was a tendency for the lithogenic diet (Figure 4B) to accelerate intestinal transit slightly compared with chow diet (Figure 4A), the differences between diets did not reach statistical significance. Our results thus affirm that, despite being fed the lithogenic diet, CCK-1R $\mathrm{R}^{-/}$mice (geometric center $=9.1 \pm 1.5$ ) continued to display significantly $(P<0.01)$ slower small-intestinal transit times than the WT mice (geometric center $=13.3 \pm 2.0$ ).

\section{Discussion}

Cholesterol gallstone formation is associated with biliary cholesterol supersaturation, rapid cholesterol crystallization through several intermediate steps, and gallbladder stasis (9). Recent progress in understanding the molecular genetic (32) and physicochemical $(28,33)$ basis of biliary cholesterol hypersecretion and cholesterol crystallization has provided many new insights into the complex pathophysiological mechanisms involved in murine cholesterol gallstone formation. The present study in a new murine model highlights two important issues: (a) gallbladder size and smallintestinal motility are, in part, mediated by CCK-1R-induced signaling, and (b) the CCK-1R control of small-intestinal transit times is a physiological response that seems to be an important influence in modulating intestinal cholesterol absorption. With targeted disruption of the Cck-1r gene, these physiological processes are altered and, as a result, susceptibility to cholesterol gallstone formation is enhanced markedly.
It has been observed in rodents that CCK inhibits gastric emptying (34). However, the physiological response of the small intestine to CCK has remained unclear until now. Expression of the Cck-1r gene has been found not only on smooth muscle of gallbladder and stomach, but also on smooth muscle of the small intestine (35). We observed that CCK-1 $\mathrm{R}^{-/-}$mice displayed significantly slower smallintestinal transit times compared with WT mice, whether ingesting chow or the lithogenic diet. Compared with the chow diet, the lithogenic diet accelerated small-intestinal transit times slightly in both strains of mice. Because small-intestinal transit time in mice is not influenced by feeding large amounts of dietary cholesterol or cholic acid (36), such an alteration could only be induced by fatty acids derived from digestion of dietary triglycerides in the lithogenic diet. Nonetheless, our results indicate that a physiologically relevant mechanism mediated by CCK-1R regulates small-intestinal motility appreciably. Furthermore, a retardation of small-intestinal transit time enhances cholesterol absorption from the intestine (Table 2 , Figure 3), most likely because of a longer residence time of the sterol in the small-intestinal lumen. This, in turn, would increase cholesterol's incorporation into mixed micelles and also would promote partitioning of cholesterol monomers out of micelles, rendering them available for intestinal capture by cholesterol influx transporter(s) on apical membranes of small-intestinal enterocytes (37). This suggests that the transit rate of cholesterol molecules throughout the small intestine is a crucial and, in health, a normal physiological step in the regulation of cholesterol absorption. Surprisingly, this concept has not been readily appreciated, although it was confirmed in humans over two decades ago (21). In the present study, we prove that CCK and its CCK-1R regulate intestinal cholesterol absorption physiologically in an animal model. Moreover, dysfunction of this receptor has deleterious effects on biliary cholesterol solubility because it leads to the secretion into bile of larger amounts of cholesterol. In earlier work we found that the percentage of cholesterol absorption decreased progressively with increases in dietary cholesterol content over the examined range of $0.02-2 \%$ by weight (31). This significant negative relationship was secondary to the pronounced effect of dietary cholesterol diluting the trace mass of radioisotope in the upper small-intestinal lumen $(27,38)$. Therefore, in the present study we used a highly precise and validated cholesterol balance method (31) to examine cholesterol absorption efficiency in both CCK-1 $\mathrm{R}^{-/-}$and WT mice while they were ingesting the lithogenic diet. We found that $\mathrm{CCK}-1 \mathrm{R}^{-/-}$mice displayed significantly higher intestinal cholesterol absorption efficiency compared with WT mice, whether mice were ingesting chow or the lithogenic diet (see Table 2). Moreover, we show that the cholesterol mass absorbed from the intestine, which reaches the liver through the chylomicron remnant pathway (39), must have in turn enhanced biliary cholesterol secretion and thereby induced biliary cholesterol supersaturation in our CCK-1R-/- mice. We reported in abstract form (40) that efficiency of intestinal cholesterol absorption actually correlates positively with prevalence of cholesterol gallstones in a large series of inbred mouse strains fed the lithogenic diet. These concepts suggest, therefore, that high intestinal cholesterol absorption efficiency is an independent pathophysiological risk factor for murine cholesterol gallstone formation.

Although it has been suggested in the past (41-44) that motility of the small bowel as well as the large bowel is sluggish in cholesterol gallstone patients, the pathogenetic mechanisms for the putative role of these conditions in cholelithogenesis are not known. In these earlier studies the authors focused on the role of the large intestine 
in cholelithogenesis, and so their hypothesis differs fundamentally from our observations in the CCK-1 $\mathrm{R}^{-/}$mouse model. The authors of these human studies (41-44) proposed that prolonged largebowel transit time, together with greater numbers of anaerobes displaying $7 \alpha$-dehydroxylation activity, promote increased formation and absorption of deoxycholic acid from the large intestine (41-44). Increased amounts of deoxycholate conjugates in bile are believed to be contributory to cholesterol gallstone formation (45) because they promote biliary cholesterol hypersecretion as well as accelerating cholesterol crystallization in gallbladder bile. However, in our studies we failed to find any differences in composition of biliary bile salt species between CCK-1R $\mathrm{R}^{-/}$and WT mice (see Results). Moreover, we reported elsewhere (46) that feeding $0.5 \%$ deoxycholic acid increased biliary deoxycholate pools in male C57L/J mice by $27 \%$ compared with the typical levels on chow (3.4\%), but small-intestinal motility was not affected (46). Interestingly, in none of the aforementioned human studies was the possibility of increased cholesterol absorption by a sluggish small-intestinal transit addressed, nor was any allusion made to the possibility that increased cholesterol absorption from the intestine promotes the formation of lithogenic bile.

Impairment of gallbladder emptying in cholesterol gallstone patients $(8,9)$ is not the result of the physical presence of gallstones per se, since the defect does not correlate with the size or number of stones, and lithotripsy-induced gallstone ablation does not reverse the gallbladder motility defect (47). Gallbladder contraction in response to i.v. CCK administration in cholesterol gallstone patients is diminished compared with the response of normal subjects to the same treatment (48), suggesting that some gallstone patients might have impaired gallbladder CCK-1R function as well as reduced CCK-1R number and/or CCK-binding capacity to CCK-1Rs $(49,50)$. Because there is impaired smooth muscle contractility in cell isolates from human gallbladders and from animal models with cholesterol gallstones, Behar and colleagues (51-53) have proposed that the gallbladder contractile mechanisms involve CCK-1R-mediated activation of phospholipase $\mathrm{C}$, leading to signal-transduction decoupling when the sarcolemmas of the smooth muscle cells of the gallbladder are highly enriched in cholesterol. This may be attributed to a cholesterol-dependent decrease in the number of or dysfunction in CCK-1Rs of gallbladder smooth muscle (52), leading to impaired contractility. A likely possibly is that this results from insertion of cholesterol molecules absorbed from bile within caveolin rafts of sarcolemmal membranes. It is apparent from the present work that a similar phenomenon may be active in the CCK-1 $\mathrm{R}^{-/}$mice. As can be inferred from Table 1 , there is a 0.26 -unit difference in mean CSI between hepatic and gallbladder bile specimens in CCK-1 $\mathrm{R}^{-/-}$mice compared with a smaller difference in mean CSI of 0.14 in WT mice. This is consistent with the possibility that, because of gallbladder stasis, biliary cholesterol molecules are absorbed in excess from gallbladders of CCK-1 $\mathrm{R}^{-1-}$ compared with the situation in WT mice. Our results therefore support the concept (51-53) that the crucial CCK-1R signal-transduction pathways may become yet more dysfunctional via the excess absorbed biliary cholesterol molecules in CCK-1 $\mathrm{R}^{-/-}$mice, thus compromising gallbladder contractility and promoting cholelithogenesis. In parallel with the findings in humans, our results substantiate the concepts that the absence of CCK-1R in mice perturbs the physiological control of gallbladder contraction as well as smallintestinal motility, leading to organ dysfunction with absorption of excess cholesterol from the lumina of both organs. The scenario for cholesterol gallstone formation in $\mathrm{CCK}-1 \mathrm{R}^{-/}$is propagated by a vicious cycle, since bile is supersaturated with cholesterol as a result of cholesterol absorption from the small intestine, and therefore gallbladder function is in turn further compromised by absorbed cholesterol molecules from cholesterol-supersaturated bile within its lumen. Hence, the CCK-1R-null mouse provides an excellent animal model for the study of cholesterol gallstone formation as promoted by these myodysfunctional mechanisms. Furthermore, the model can be systematically evaluated to investigate principles underlying gallbladder and small-intestinal contractile functions in cholelithogenesis, at both the molecular and the cellular levels.

In summary, ablation of the Cck-1r gene impairs small-intestinal transit in mice whether chow or a lithogenic diet is ingested; this impairment in turn leads to increased intestinal cholesterol absorption, which results in enhanced biliary cholesterol secretion. Absence of functional CCK-1Rs also increases gallbladder size and cholesterol absorption from bile, which further impairs muscle contractility and probably results in profound gallbladder stasis (54). These dysfunctions of gastrointestinal motility contribute to cholesterol gallstone formation by facilitating more efficient intestinal cholesterol absorption and biliary cholesterol hypersecretion, leading to crystallization and growth, as well as agglomeration of solid-cholesterol crystals in gallbladders that have become "large, lax and lazy" (55). These findings, taken together, indicate that prolonged small-intestinal transit times, increased cholesterol absorption, and gallbladder hypomotility are important interdependent and conflating risk factors for cholesterol gallstone formation in this animal model.

\section{Methods}

Chemicals. Intralipid (20\%, wt/vol) was purchased from Pharmacia (Clayton, North Carolina, USA), and medium-chain triglyceride was obtained from Mead Johnson Nutritionals (Evansville, Indiana, USA). Radioisotopes $\left[1,2-{ }^{3} \mathrm{H}\right]$ cholesterol and $\left[4-{ }^{-14} \mathrm{C}\right]$ cholesterol were purchased from NEN Life Science Products Inc. (Boston, Massachusetts, USA), and [5,6- $\left.{ }^{3} \mathrm{H}\right]$ sitostanol was obtained from American Radiolabeled Chemicals Inc. (St. Louis, Missouri, USA). For HPLC of biliary lipids, all reagents were spectra-analyzed HPLC grade from Fisher Scientific Co. (Fair Lawn, New Jersey, USA).

Animals and diets. Generation of CCK-1 $\mathrm{R}^{-/-}$mice and general phenotypic properties have been reported previously (22). The CCK-A receptor is now referred to as CCK-1R (official gene symbol is Cck-1r) (56). We studied male homozygous CCK-1R knockout mice and WT mice of the same 129/SvEv background (Charles River Laboratories, Wilmington, Massachusetts, USA) at 3-6 months of age. All animals were maintained in a temperature-controlled room $\left(22 \pm 1^{\circ} \mathrm{C}\right)$ with 12 -hour light ( 6 am- 6 pm) cycles. Mice were allowed free access to water and standard Purina rodent chow (Purina Mills Inc., St. Louis, Missouri, USA), which contains trace quantities $(<0.02 \%)$ of cholesterol (27). During gallstone-induction experiments, animals were fed a semi-synthetic lithogenic diet (28) containing $1 \%$ cholesterol, $0.5 \%$ cholic acid, and $15 \%$ butterfat for 12 weeks. All experiments were executed according to accepted criteria for the care and experimental use of laboratory animals, and euthanasia was consistent with recommendations of the American Veterinary Medical Association. Ethical protocols for animal experimentation were approved by the Institutional Animal Care and Research Committees of Harvard Medical School and Tufts-New England Medical Center, both of Boston, Massachusetts, USA.

Collection and microscopic analysis of gallbladder bile specimens and gallstones. After 12 weeks on the lithogenic diet, the WT mice $(n=26)$ and CCK-1R-1mice $(n=37)$ were fasted overnight but allowed free access to water. Animals were weighed and anesthetized with an intraperitoneal injection of 35 $\mathrm{mg} / \mathrm{kg}$ pentobarbital (Abbott Laboratories, North Chicago, Illinois, USA). After cholecystectomy (28), gallbladder volume was measured by weighing the whole gallbladder and numerically equating gallbladder weight with 
gallbladder volume (28). Gallbladders were then opened at the fundus, and $5 \mu \mathrm{l}$ of fresh gallbladder bile were digitally expressed and examined by direct and polarized light microscopy for mucin gel, liquid crystals, cholesterol crystals, and stones according to previously established criteria (28). Pooled gallbladder bile samples were ultracentrifuged at $100,000 \mathrm{~g}$ for 30 minutes at $37^{\circ} \mathrm{C}$ and filtered through a preheated $\left(37^{\circ} \mathrm{C}\right)$ Swinnex-GS filter $(0.22$ $\mu \mathrm{m}$ ) assembly (Millipore Corp., Bedford, Massachusetts, USA). Samples were then frozen and stored at $-20^{\circ} \mathrm{C}$ for further lipid analysis (see later).

Collection of hepatic bile specimens and measurement of circulating bile salt pool sizes. Biliary lipid secretory studies (33) were carried out on additional groups of CCK- $1 \mathrm{R}^{-/-}$and WT mice ( $n=6$ per group) fed the lithogenic diet for 12 weeks. In brief, after caudad ligation, the common bile duct was cannulated with a polyethylene catheter (PE-10). After observation of bile fistula flow, the cystic duct was doubly ligated and a cholecystectomy was performed. Hepatic bile was collected under gravity drainage every hour for 8 hours. Each collection was examined by direct and polarized light microscopy, volumes were determined, and samples were frozen and stored at $-20^{\circ} \mathrm{C}$ for further lipid analyses (see later). For measurement of the circulating bile salt pool sizes, 8-hour biliary washout studies in mice were performed according to published methods (33). During surgery, mouse body temperature was maintained at $37 \pm 0.5^{\circ} \mathrm{C}$ with a heating lamp and monitored with a thermometer. Animals were kept lightly anesthetized with an intraperitoneal injection of $17 \mathrm{mg} / \mathrm{kg}$ pentobarbital every 2 hours, and $100 \mu \mathrm{l}$ of $0.9 \% \mathrm{NaCl}$ was given hourly via the abdominal cavity to maintain hydration.

Determination of intestinal cholesterol absorption by the plasma dual-isotope ratio method. Cholesterol absorption was measured by a plasma dual-isotope ratio method $(27,30,31)$ in chow-fed CCK-1 $\mathrm{R}^{-/-}$and WT mice ( $n=14$ per group). Each animal was injected i.v. with $100 \mu \mathrm{l}$ of Intralipid containing $2.5 \mu \mathrm{Ci}$ $\left[{ }^{3} \mathrm{H}\right]$ cholesterol; immediately thereafter we administered an intragastric dose of $150 \mu \mathrm{l}$ of medium-chain triglyceride in which $1 \mu \mathrm{Ci}$ of $\left[{ }^{14} \mathrm{C}\right]$ cholesterol was dissolved. To determine the ratio of $\left[{ }^{14} \mathrm{C}\right]$ cholesterol and $\left[{ }^{3} \mathrm{H}\right]$ cholesterol in plasma at exactly 3 days, $100-\mu \mathrm{l}$ aliquots of plasma and the original dosing mixture were mixed individually with $10 \mathrm{ml}$ of EcoLite (ICN Biomedicals Inc., Costa Mesa, California, USA) and counted in a liquid scintillation spectrometer (Beckman Instruments Inc., San Ramon, California, USA). The radioisotope ratio in plasma was used for calculating the percentage cholesterol absorption as follows: Percentage cholesterol absorption $=$ (percentage of intragastric dose of $\left[{ }^{14} \mathrm{C}\right]$ cholesterol per milliliter plasma/percentage of i.v. dose of $\left[{ }^{3} \mathrm{H}\right]$ cholesterol per milliliter plasma) $\times 100$.

Measurement of cholesterol absorption by cholesterol balance methodology. Because large amounts of dietary cholesterol in the upper small intestine dilute the specific activity of radioisotopic cholesterol, we used cholesterol balance methodology (31) to analyze cholesterol absorption efficiency in WT and CCK-1 $\mathrm{R}^{-/-}$mice during feeding of the lithogenic diet as well as in control groups of mice ingesting chow. Mice were housed in individual metabolic cages with wire-mesh bottoms and allowed to adapt to the environment for 2 weeks. When BW, food ingestion, and daily fecal excretion became constant (i.e., an apparent metabolic steady state), food intake was measured and feces were collected daily for 7 days. For each balance study, CCK- $1 \mathrm{R}^{-/-}$and WT mice ( $n=7$ per group) were fed for 7 days with chow or the lithogenic diet. After we had followed this protocol rigorously, we anesthetized the animals with pentobarbital, performed a cholecystectomy, cannulated the common bile duct with a PE-10 catheter, and collected hepatic bile for 1 hour. Biliary and dietary cholesterol contents were measured by HPLC. After saponifying and extracting fecal neutral steroids, they were assayed by HPLC and the percentage cholesterol absorption was calculated according to a validated sterol balance method for mice (31).

Measurement of small-intestinal transit times. Small-intestinal transit times in CCK- $1 \mathrm{R}^{-/-}$and WT mice on chow ( $n=13$ per group), as well as in both groups on the lithogenic diet for 14 days ( $n=5$ per group), respectively, were measured according to described methods (30). Under pentobarbital anesthesia, $2 \mu \mathrm{Ci}$ of $\left[{ }^{3} \mathrm{H}\right]$ sitostanol - as a nonabsorbable reference marker, dissolved in $100 \mu \mathrm{l}$ of medium-chain triglyceride - was instilled into the small intestine of mice via a previously fitted in situ externalized duodenal catheter (30). Exactly 30 minutes after instillation, mice were again anesthetized with an intraperitoneal injection of $35 \mathrm{mg} / \mathrm{kg}$ pentobarbital. The abdomen was opened, and stomach, small and large intestines, and cecum were removed rapidly while avoiding digital or instrumental compression. The small intestine was frozen promptly in liquid $\mathrm{N}_{2}$, placed on a $50-\mathrm{cm}$ ruled template, and cut into 20 equal segments with a scalpel blade. Individual segments were placed in tubes containing $10 \mathrm{ml}$ of $\mathrm{CHCl}_{3}-\mathrm{CH}_{3} \mathrm{OH}$ (2:1, vol/vol), homogenized, and centrifuged at $10,000 \mathrm{~g}$ for 30 minutes. The samples were then stored at $4{ }^{\circ} \mathrm{C}$ for 48 hours. Well-mixed portions $(1 \mathrm{ml})$ were pipetted into counting vials, and the solvent was evaporated under $\mathrm{N}_{2}$. EcoLite $(7 \mathrm{ml})$ was then added, and radioactivity was determined by liquid scintillation counting. Samples of stomach, cecum, and large intestine were also analyzed, but none showed appreciable radioactivity above background. Using these data, a calculation of small-intestinal transit time was carried out by two arithmetic methods (30): (a) the percentages of total $\left[{ }^{3} \mathrm{H}\right]$ sitostanol radioactivity in each of the 20 small-intestinal segments were transformed to cumulative percentages passing each segment; (b) the geometric center for the distribution of radioactivity within the small intestine was derived from the sum of the proportions of $\left[{ }^{3} \mathrm{H}\right]$ sitostanol per segment multiplied by segment number.

Biliary lipid analyses. Biliary phospholipids were analyzed by an inorganic phosphorus method (57). Total and individual bile salt concentrations were measured by HPLC (28). Biliary cholesterol as well as cholesterol content in chow and gallstones was determined by HPLC (28). CSIs of gallbladder and hepatic bile samples were calculated from critical tables (23). Relative lipid compositions of mouse gallbladder and hepatic bile samples were plotted on triangular phase diagrams according to their rounded-off mean total lipid concentrations (24). Phase boundaries and crystallization pathways were extrapolated from model bile systems based on sodium taurocholate at $37^{\circ} \mathrm{C}(24,25)$. Hydrophobicity indexes of hepatic bile were calculated according to Heuman's method (29).

Statistical analyses. All data are expressed as mean \pm SD. Differences among groups of mice were assessed for statistical significance by Student's $t$ test, Mann-Whitney $U$ test, or $\chi^{2}$ test. Analyses were performed with SuperANOVA software (Abacus Concepts Inc., Berkeley, California, USA). Statistically significant differences were defined as a two-tailed probability of less than 0.05 .

\section{Acknowledgments}

We are greatly indebted to Helen H.-F. Wang (Beth Israel Deaconess Medical Center, Boston, Massachusetts, USA) for excellent technical assistance. D.Q.-H. Wang is a recipient of a New Scholar Award from the Ellison Medical Foundation (19992003). This work was supported in part by the Ellison Medical Foundation (to D.Q.-H. Wang), as well as research and center grants DK54012 (to D.Q.-H. Wang), DK36588, DK34854, DK52911 (to M.C. Carey), DK46767 (to A.S. Kopin), and DK34928 (GRASP), all from the NIH (US Public Health Service). A.S. Kopin is a Tufts New England Medical Center Molecular Cardiology Research Institute investigator.

Received for publication August 30, 2002, and accepted in revised form June 29, 2004.

Address correspondence to: Martin C. Carey, Gastroenterology Division, Brigham and Women's Hospital, 75 Francis Street, Boston, Massachusetts 02115, USA. Phone: (617) 732-5822; Fax: (617) 730-5807; E-mail: mccarey@rics.bwh.harvard.edu. 
1. Liddle, R.A. 1994. Cholecystokinin. In Gut peptides. J.H. Walsh and G.J. Dockray, editors. Raven. New York, New York, USA. 175-216.

2. Wank, S.A. 1995. Cholecystokinin receptors. Am. J Physiol. 269:G628-G646.

3. Grider, J.R. 1994. Role of cholecystokinin in the regulation of gastrointestinal motility. J. Nutr. 124:1334S-1339S

4. Wank, S.A., Pisegna, J.R., and De Weerth, A. 1994. Cholecystokinin receptor family. Molecular cloning, structure, and functional expression in rat, guinea pig, and human. Ann. N. Y. Acad. Sci. 713:49-66.

5. Behar, J., Lee, K.Y., Thompson, W.R., and Biancani, P. 1989. Gallbladder contraction in patients with pigment and cholesterol stones. Gastroenterology. 97:1479-1784

6. Pomeranz, I.S., and Shaffer, E.A. 1985. Abnormal gallbladder emptying in a subgroup of patients with gallstones. Gastroenterology. 88:787-791.

7. Portincasa, P., et al. 2000. Gallbladder motility and cholesterol crystallization in bile from patients with pigment and cholesterol gallstones. Eur. J. Clin. Invest. 30:317-324.

8. LaMorte, W.W., Schoetz, D.J., Birkett, D.H., and Williams, L.F. 1979. The role of the gallbladder in the pathogenesis of cholesterol gallstones. Gastroenterology. 77:580-592.

9. Afdhal, N.H., and Carey, M.C. 1995. New perspectives on gallstone pathogenesis and prevention. J. Ir. Coll. Physicians Surg. 24:267-278.

10. Everson, G.T., McKinley, C., Lawson, M., Johnson, M., and Kern, F., Jr. 1982. Gallbladder function in the human female: effect of the ovulatory cycle, pregnancy, and contraceptive steroids. Gastroenterology. 82:711-719.

11. Braverman, D.Z., Johnson, M.L., and Kern, F. Jr., 1980. Effects of pregnancy and contraceptive steroids on gallbladder function. N. Engl. J. Med. 302:362-364

12. Maringhini, A., et al. 1987. Sludge and stones in gallbladder after pregnancy: prevalence and risk factors. J. Hepatol. 5:218-223.

13. Maringhini, A., Ciambra, M., Barcelliere, P., Raimondo, M., and Pagliaro, L. 1988. Sludge, stones and pregnancy. Gastroenterology. 95:1160-1161.

14. Thompson, J.C., et al. 1982. Correlation between release of cholecystokinin and contraction of the gallbladder in patients with gallstones. Ann. Surg. 195:670-676

15. Griffen, W.O., et al. 1980. Cholecystokinin cholecystography in the diagnosis of gallbladder disease. Ann. Surg. 191:636-640.

16. Doty, J.E., Pitt, H.A., Kuchenbecker, S.L., and DenBesten, L. 1983. Impaired gallbladder emptying before gallstone formation in the prairie dog. Gastroenterology. 85:168-174.

17. Pellegrini, C.A., Ryan, T., Broderick, W., and Way, L.W. 1986. Gallbladder filling and emptying during cholesterol gallstone formation in the prairie dog: a cholescintigraphic study. Gastroenterology. 90:143-149.

18. Fridhandler, T.M., Davison, J.S., and Shaffer, E.A. 1983. Defective gallbladder contractility in the ground squirrel and prairie dog during the early stages of cholesterol gallstone formation. Gastroenterology. 85:830-836.

19. Behar, J., Rhim, B.Y., Thompson, W., and Biancani, P. 1993. Inositol trisphosphate restores impaired human gallbladder motility associated with cholesterol stones. Gastroenterology. 104:563-568.

20. Sitzmann, J.V., Pitt, H.A., Steinborn, P.A., Pasha, Z.R., and Sanders, R.C. 1990. Cholecystokinin prevents parenteral nutrition induced biliary sludge in humans. Surg. Gynecol. Obstet. 170:25-31.

21. Ponz de Leon, M., et al. 1982. Influence of smallbowel transit time on dietary cholesterol absorp- tion in human beings. N. Engl. J. Med. 307:102-103.

22. Kopin, A.S., et al. 1999. The cholecystokinin-A receptor mediates inhibition of food intake yet is not essential for the maintenance of body weight. J. Clin. Invest. 103:383-391.

23. Carey, M.C. 1978. Critical tables for calculating the cholesterol saturation of native bile. J. Lipid Res. 19:945-955.

24. Wang, D.Q.-H., and Carey, M.C. 1996. Complete mapping of crystallization pathways during cholesterol precipitation from model biles: influence of physical-chemical variables of pathophysiologic relevance and identification of a stable liquid crystalline state in cold, dilute and hydrophilic bile salt-containing systems. J. Lipid Res. 37:606-630.

25. Carey, M.C., and Small, D.M. 1978. The physical chemistry of cholesterol solubility in bile. Relationship to gallstone formation and dissolution in man. J. Clin. Invest. 61:998-1026.

26. Wang, D.Q.-H., Cohen, D.E., Lammert, F., and Carey, M.C. 1999. No pathophysiologic relationship of soluble biliary proteins to cholesterol crystallization in human bile. J. Lipid Res. 40:415-425.

27. Wang, D.Q.-H., Lammert, F., Cohen, D.E., Paigen, B., and Carey, M.C. 1999. Cholic acid aids absorption, biliary secretion, and phase transitions of cholesterol in murine cholelithogenesis. Am. J. Physiol. 276:G751-G760.

28. Wang, D.Q.-H., Paigen, B., and Carey, M.C. 1997. Phenotypic characterization of Lith genes that determine susceptibility to cholesterol cholelithiasis in inbred mice: physical-chemistry of gallbladder bile. J. Lipid Res. 38:1395-1411.

29. Heuman, D.M. 1989. Quantitative estimation of the hydrophilic-hydrophobic balance of mixed bile salt solutions. J. Lipid Res. 30:719-730.

30. Wang, D.Q.-H., Paigen, B., and Carey, M.C. 2001. Genetic factors at the enterocyte level account for variations in intestinal cholesterol absorption efficiency among inbred strains of mice. J. Lipid Res. 42:1820-1830

31. Wang, D.Q.-H., and Carey, M.C. 2003. Measurement of intestinal cholesterol absorption by plasma and fecal dual-isotope ratio, mass balance, and lymph fistula methods in the mouse: an analysis of direct versus indirect methodologies. J. Lipid Res. 44:1042-1059.

32. Lammert, F., Carey, M.C., and Paigen, B. 2001. Chromosomal organization of candidate genes involved in cholesterol gallstone formation: a murine gallstone map. Gastroenterology. 120:221-238.

33. Wang, D.Q.-H., Lammert, F., Paigen, B., and Carey, M.C. 1999. Phenotypic characterization of Lith genes that determine susceptibility to cholesterol cholelithiasis in inbred mice: pathophysiology of biliary lipid secretion. J. Lipid Res. 40:2066-2079.

34. Raybould, H.E., and Tache, Y. 1998. Cholecystokinin inhibits gastric motility and emptying via a capsaicin-sensitive vagal pathway in rats. Am. J. Physiol. 255:G242-G246.

35. Lacourse, K.A., Lay, J.M., Swanberg, L.J., Jenkins, C., and Samuelson, L.C. 1997. Molecular structure of the mouse CCK-A receptor gene. Biochem. Biophys. Res. Commun. 236:630-635.

36. Wang, D.Q.-H., Tazuma, S., Cohen, D.E., and Carey, M.C. 2003. Feeding natural hydrophilic bile acids inhibits intestinal cholesterol absorption: studies in the gallstone-susceptible mouse. Am. J. Physiol. 285:G494-G502.

37. Altmann, S.W., et al. 2004. Niemann-Pick C1 Like 1 protein is critical for intestinal cholesterol absorption. Science. 303:1201-1204.

38. Duan, L.-P., Wang, H.H., and Wang, D.Q.-H. 2004 Cholesterol absorption is mainly regulated by the jejunal and ileal ATP-binding cassette sterol efflux transporters $\mathrm{Abcg} 5$ and $\mathrm{Abcg} 8$ in mice.
J. Lipid Res. 45:1312-1323.

39. Wang, D.Q.-H., and Zhang, L.-N. 2000. Dietary cholesterol through chylomicron pathway enhances murine cholelithogenesis [abstract]. Gastroenterology. 118:A715.

40. Wang, D.Q.-H., Paigen, B., and Carey, M.C. 1998. Genetic variations in cholesterol absorption efficiency are associated with cholesterol gallstone formation in inbred mice [abstract]. Hepatology. 28:163A.

41. Heaton, K.W., Emmett, P.M., Symes, C.L., and Braddon, F.E. 1993. An explanation for gallstones in normal-weight women: slow intestinal transit. Lancet. 341:8-10.

42. Shoda, J., et al. 1995. Increase of deoxycholate in supersaturated bile of patients with cholesterol gallstone disease and its correlation with de novo syntheses of cholesterol and bile acids in liver, gallbladder emptying, and small intestinal transit. Hepatology. 21:1291-1302.

43. Azzaroli, F., et al. 1999. Sluggish small bowel motility is involved in determining increased biliary deoxycholic acid in cholesterol gallstone patients. Am. J. Gastroenterol. 94:2453-2459.

44. Thomas, L.A., et al. 2000. Mechanism for the transit-induced increase in colonic deoxycholic acid formation in cholesterol cholelithiasis. Gastroenterology. 119:806-815.

45. Marcus, S.N., and Heaton, K.W. 1986. Intestinal transit, deoxycholic acid and the cholesterol saturation of bile-three inter-related factors. Gut. 27:550-558.

46. Wang, D.Q.-H., Tazuma, S., Cohen, D.E., and Carey, M.C. 2003. Feeding natural hydrophilic bile acids inhibits intestinal cholesterol absorption: studies in the gallstone-susceptible mouse. Am. J. Physiol. 285:G494-G502.

47. Spengler, U., Sackmann, M., Sauerbruch, T., Holl, J., and Paumgartner, G. 1989. Gallbladder motility before and after extracorporeal shock-wave lithotripsy. Gastroenterology. 96:860-863.

48. Northfield, T.C., et al. 1980. Gall-bladder sensitivity to cholecystokinin in patients with gallstones. Br. Med. J. 280:143-144.

49. Miller, L.J., Holicky, E.L., Ulrich, C.D., and Wieben, E.D. 1995. Abnormal processing of the human cholecystokinin receptor gene in association with gallstones and obesity. Gastroenterology. 109:1375-1380.

50. Schneider, H., Sanger, P., and Hanisch, E. 1997. In vitro effects of cholecystokinin fragments on human gallbladders. Evidence for an altered CCKreceptor structure in a subgroup of patients with gallstones. J. Hepatol. 26:1063-1068.

51. Yu, P., et al. 1995. Direct $G$ protein activation reverses impaired CCK signaling in human gallbladders with cholesterol stones. Am. J. Physiol. 269:G659-G665.

52. Xiao, Z.L., et al. 1999. CCK receptor dysfunction in muscle membranes from human gallbladders with cholesterol stones. Am. J. Physiol. 276:G1401-G1407.

53. Yu, P., et al. 1998. Signal transduction pathways mediating CCK-induced gallbladder muscle contraction. Am. J. Physiol. 275:G203-G211.

54. Suzuki, S., et al. 2001. Importance of CCK-A receptor for gallbladder contraction and pancreatic secretion: a study in CCK-A receptor knockout mice. Jpn. J. Physiol. 51:585-590.

55. Shaffer, E.A. 1992. Abnormalities of gallbladder function in cholesterol gallstone disease: bile and blood, mucosa and muscle - the list lengthens. Gastroenterology. 102:1808-1812.

56. Noble, F., et al. 1999. International Union of Pharmacology. XXI. Structure, distribution, and functions of cholecystokinin receptors. Pharmacol. Rev. 51:745-781.

57. Bartlett, G.R. 1959. Phosphorous assay in column chromatography. J. Biol. Chem. 234:466-468. 